\title{
La configuración de la enseñanza de la biología: una inquietud por la pedagogía
}

Paola Andrea Roa García ${ }^{1}{ }^{10}$

Universidad Pedagógica Nacional - Colombia

Autor de correspondencia: ${ }^{1}$ proa@pedagogica.edu.co Recibido: 31 de marzo de 2020 Revisado: 30 de mayo de 2020 Aprobado: 28 de julio de 2020 Publicado: 28 de septiembre de 2020

\section{Resumen}

Se presentan algunos nodos problémicos en la configuración de la enseñanza de la biología, que posibilitan interrogar el lugar de la pedagogía en su devenir en la historia de la escuela colombiana, con el propósito de contribuir a la constitución actual de su objeto. Una mirada genealógica al archivo -1890-2016- permite decir que la biología emerge en la escuela colombiana en la primera mitad del siglo pasado a propósito de condiciones relacionadas con la educación y los fines socioculturales. Su enseñanza ha estado vinculada con hacer ciencia en la escuela y se ha relacionado con las ciencias naturales - en cuanto a las nociones de integración e interdisciplinariedad- $y$, en la actualidad, con su desplazamiento a propósito de la educación ambiental y la interculturalidad. Estas se proponen como nodos que señalan la ausencia de la pedagogía en su configuración o su reducción a las ciencias de la educación, al intento de reproducción de la ciencia biológica y a la didáctica, entendida como las formas de hacer ciencia en la escuela.

Palabras claves: educación, enseñanza de las ciencias, historia de la educación, escuela

\section{(c) $)(1) \Theta \Theta$}




\title{
The configuration of biology teaching: a concern for pedagogy
}

\begin{abstract}
Some problematic nodes in the configuration of biology teaching are presented, which allow us to question the place of pedagogy in its journey through the history of the Colombian school, in order to contribute to the current constitution of its subject of matter. A genealogical look at the archive-1890-2016-allows us to state that biology emerged in the Colombian school in the first half of the last century due to conditions related to education and socio-cultural purposes. Its teaching has been linked to doing science in school and has been related to the natural sciences-in terms of the notions of integration and interdisciplinarity - and currently to its displacement about environmental education and interculturality. These are proposed as nodes that point to the absence of pedagogy in its configuration or its reduction to the sciences of education, to the attempt to reproduce biological science and to didactics - understood as the ways of doing science in school.
\end{abstract}

Keywords: education, science teaching, history of education, school

\section{A configuração do ensino da biologia: uma preocupação pela pedagogia}

\section{Resumo}

Apresentam-se alguns nós problemáticos na configuração do ensino da biologia, que possibilitam interrogar o lugar da pedagogia em sua evolução na história da escola colombiana, com o propósito de contribuir para a constituição atual de seu objeto. Um olhar genealógico sobre o arquivo - 1890-2016- permite-nos dizer que a biologia surgiu na escola colombiana na primeira metade do século passado a propósito das condições relacionadas com a educação e aos fins socioculturais. O seu ensino tem estado ligado ao fazer ciência na escola e tem estado relacionado com as ciências naturais - em termos das noções de integração e interdisciplinaridade- e, hoje, com a sua deslocação em relação à educação ambiental e à interculturalidade. Estas são propostas como nós que indicam a ausência da pedagogia na sua configuração ou sua redução às ciências da educação, à tentativa de reprodução da ciência biológica e à didática, entendida como as formas de fazer ciência na escola.

Palavras-chave: educação, ensino das ciências, história da educação, escola

\section{Una inquietud por la pedagogía en la enseñanza de la biología ${ }^{1}$}

La inquietud por el lugar de la pedagogía en la enseñanza de la biología implica la mirada a su emergencia y a las relaciones que le han dado lugar. De ese modo, el rastreo de prácticas permite visibilizar las condiciones de posibilidad de tales relaciones. Desde tal perspectiva,

1. Escrito resultado de la investigación en curso Aprendizaje y vida. Prácticas de formación contemporáneas, proyecto de tesis doctoral realizado en el Doctorado en Educación de la Universidad Pedagógica Nacional. 
el propósito de este artículo es presentar algunos nodos ${ }^{2}$ problémicos en la configuración de la enseñanza de la biología, que posibilitan plantear algunas sospechas sobre el lugar de la pedagogía en su devenir.

La enseñanza de la biología es un objeto de interrogación no acabado, ni inmutable, pues, a partir de la investigación, es posible decir que no siempre se ha hablado de ella y que las prácticas han cambiado a propósito de condiciones singulares, azarosas, discontinuas e incluso contradictorias. Así, visibilizar sus relaciones con la pedagogía posibilita la mirada a la irrupción, al devenir y al desplazamiento de la enseñanza de la biología (Roa et al., 2018).

La conformación de un archivo, más allá de presentar una cronología, pretende señalar nodos problémicos para pensar la enseñanza de la biología desde la pedagogía. Así, la historia no es un método, sino una forma de alejarse de lo normal, de lo establecido, para con extrañeza, interrogar la naturalización que impera sobre las prácticas de enseñanza y la formación en biología. La configuración interroga las condiciones de posibilidad de las prácticas que se llevan a cabo en la escuela, donde están inmersas las relaciones de fuerza, las formas de saber y la subjetividad. Es recurrente su relación con la educación y el aprendizaje.

La educación y el aprendizaje aparecen como sistema que fija el campo de acción de la escuela. Los discursos que los enmarcan permiten establecer cómo sus reglas posibilitan las prácticas, en este caso, relacionadas con la enseñanza de la biología, pues condicionan los enunciados, los objetos y los juegos teóricos que pueden y deben verse involucrados en esta.

En tal sentido, este acercamiento a la configuración de la enseñanza de la biología se lleva a cabo desde lo pedagógico, es decir, como posibilidad de pensamiento más allá de la historia lineal, "como saber y como práctica, lo pedagógico como lo que permite a los maestros entrar en relación con el conocimiento; lo pedagógico como acontecimiento social susceptible de ser descrito en su especificidad histórica" (Zuluaga, 1999, p. 17). Por lo tanto, no se asume la pedagogía como educación ni como aprendizaje, sino como la relación entre estos, desde la enseñanza como objeto de saber.

A continuación, se muestra una ruta de lectura de lo que el archivo conformado visibiliza en relación con la educación, la enseñanza y el aprendizaje; lo que circula entorno a estas y sus posibles relaciones con la configuración de la enseñanza de la biología en la escuela colombiana. Es importante notar cómo la pedagogía-como relación entre educación y aprendizaje - no es parte de los discursos que confluyen en la escuela, lo cual posibilita pensar que son capturados de lo que circula y responden a discursos hegemónicos que convierten a la educación en un medio de divulgación; a la enseñanza en instrucción y aprendizaje de lo que se establece como normas que los individuos deben asumir, saber y hacer; y a la escuela en un espacio de reproducción de lo instaurado.

\section{La pedagogía en el devenir de la enseñanza de la biología}

En esta investigación se asume la pedagogía desde lo propuesto por Zuluaga et al. (2003), como un saber:

2. Nodo es entendido como noción metodológica que pone en evidencia elementos y relaciones que pueden parecer dispersos. El nodo permite acentuar la mirada y abordaje desde una perspectiva compleja y rizomática. De este modo, posibilita problematizar lo que parece normal y acabado.Para el caso, es un cruce de relaciones que posibilita visibilizar distintas formas y fuerzas de relación que dan lugar a las prácticas de la enseñanza de la biología. 
La adopción del término saber para la pedagogía busca que cualquier investigador de la educación se ubique en el conocimiento como si estuviera en un espacio abierto, desde el cual pueda desplazarse y orientarse, desde las regiones más sistematizadas hasta los espacios plurales que están en permanente intercambio entre sí. (p. 15)

Entender la pedagogía como saber posibilita comprender las prácticas en movimiento, sin ubicarse desde lo que se cree preexistente, como las ciencias, ni tampoco desde las lógicas promovidas por las condiciones sociales y políticas. La noción de saber no solo implica una conceptualización distinta, sino - sobretodo - una mirada a las prácticas desde sus condiciones de posibilidad, es decir, como forma no terminada, que no se sitúa en lo definido, sino en el cómo deviene.

De esta manera, la investigación, propuesta en el marco de los estudios doctorales, se realiza desde el saber pedagógico. No busca confirmar lo ya dicho sobre la configuración de la enseñanza de la biología, ni verificar la reproducción de la ciencia biológica, sino cómo emerge la biología en la escuela, cómo se estabiliza, a propósito de qué condiciones cambia. Se pone en cuestión "el concepto" como definición y totalidad, de allí que "la enseñanza de la biología" implique las relaciones entre conceptos e incluso su movimiento.

Así, la pedagogía ha sido desplazada, como lo mencionan Zuluaga et al.(2003), por las ciencias de la educación, pues la educación "restringe el significado, la acción y el campo del concepto enseñanza" (p. 22),lo que conlleva problemas como la desarticulación, la atomización, la subordinación y la instrumentación de la pedagogía.

Entender la enseñanza como transmisión, como gestión de conocimientos, la desplaza por las prácticas que constituyen el aprendizaje hoy, relacionados con la vida entendida como empresa:

El aprendizaje adquirió durante el último siglo un lugar central en lo que se refiere a la necesidad de disponer todos los escenarios públicos y privados para que cada individuo, sujeto de su propio aprendizaje, adquiera las competencias y habilidades necesarias para "aprender a aprender" y, así, “aprender a lo largo de la vida". (Marín, 2019, p. 106)

Al preguntar por la enseñanza de la biología y su devenir histórico, son muy pocos los trabajos que se encuentran. Muchos de ellos se refieren a las ciencias naturales que, aunque se relacionan, incluyen también otras disciplinas como la química, la física y —a principios del siglo pasado- la historia natural, la fisiología y la medicina, entre otras. Desde esa perspectiva, Not (2000) habla de una historia de las disciplinas escolares y menciona la biología animal y la biología vegetal, al señalar que el papel de la enseñanza es aportar a la cultura, desde el uso de la clasificación de los seres vivos.

Por otro lado, Avanzini (1990) propone la mirada a la enseñanza de las ciencias naturales desde las técnicas que permiten regular la limpieza y el desarrollo de los niños. Presenta una preocupación por el cuerpo desde la biopedagogía - o pedagogía fundada en el cuerpo- .

Goodson (2008), desde la historia del currículo, menciona que en la década delos 30 la biología ingresó al currículo escolar en Gran Bretaña. Muestra su transición de la marginalidad al utilitarismo, hasta ser entendida como disciplina. Estas miradas posibilitan interrogar el lugar de la pedagogía en la configuración de la enseñanza de la biología, ya que lo pedagógico es asumido a partir de otras disciplinas "científicas", donde su objeto es difuso y cualquier práctica educativa se constituye en pedagógica. 
Para el caso colombiano, se puede rastrear la emergencia de la biología en la escuela en la primera mitad del siglo pasado (Roa \& Herrera, 2010). No obstante, los trabajos alrededor de la historia de la enseñanza de la biología son escasos. Sin embargo, con la creación del departamento de Biología de la Universidad Pedagógica Nacional [UPN] en 1968, se visibiliza esta necesidad, en tanto que se propone su aporte a las ciencias de la educación:"La reforma de 1969 buscaba fundamentalmente aumentar las oportunidades de acceso a la educación superior especialmente en la formación de licenciados en Ciencias de la Educación, mejorar el empleo y el rendimiento de los recursos económicos, materiales y humanos"(UPN, 1984, p. 3).

Chona et al. (1998) proponen una relación entre el saber enseñado y la disciplina biológica. Así, la enseñanza posibilita pensamiento dentro de los lineamientos de la ciencia. Identifican cuatro momentos en la historia de la enseñanza de la biología: saberes mezclados, saberes circunscritos, ciencia biológica y profesionalización. Luego presentan una historia de la disciplina biológica y su inserción en el sistema de enseñanza.

Riveros (2006) realiza un acercamiento a la enseñanza de la biología, desde la historia de sus prácticas, a partir del reconocimiento de sus condiciones de posibilidad. Afirma que es evidente que, en lo referente a la enseñanza de la biología y de las ciencias, se hace mayor alusión a la transmisión de conocimientos — de saberes puros-, mediante el rigor empírico-positivista, así como a lo que debe hacer el maestro para esto y lo que se supone debe ser la respuesta del estudiante.

Roa y Herrera (2010) proponen la "biología en la escuela" como noción que permite distanciarse del lugar común que origina la enseñanza de la biología, el cual está relacionado con la reproducción de la ciencia biológica, el cuestionamiento de las malas prácticas de los maestros, las críticas sobre la deficiente formación en la disciplina y la didáctica desde la enseñanza psicologizada de conceptos.

Según lo expuesto, la pregunta por la pedagogía en el devenir de la enseñanza de la biología, más que remitir a formas de nombrar, posibilita visibilizar las prácticas que dejan entrever las tensiones, las posibilidades, las limitaciones y, en todo ello, el movimiento, en tanto campo abierto, no determinado y no circunscrito a las disciplinas científicas.

De esa manera, no es pertinente presentar elementos relacionados con la historia de la biología científica, pues no se pretende asumirla como homóloga de la enseñanza de la biología, pero sí es apropiado nombrar que durante mucho tiempo su estatuto científico ha estado en cuestión debido a la imposibilidad de universalizar su objeto: "lo vivo", aunque este tampoco haya permanecido estático y, en muchas ocasiones, aparezca indiferenciado de "la vida" y, en otras, relacionado con el "vivir". Sin embargo, es relevante mencionar que el estudio de la biología desde una perspectiva histórico-genealógica es una ruta pendiente por explorar.

\section{Metodología: la configuración de la enseñanza de la biología}

La mirada a la configuración de la enseñanza de la biología, desde la pedagogía, implica cuestionar varios asuntos que se han asumido como estáticos y, en muchos casos, como la 
$\operatorname{verdad}^{3}$. La configuración propone asumir la historia de otro modo: como singularidad que no responde a cronologías, ni a relaciones causa-efecto, ni mucho menos al origen; pretende desprenderse de la totalidad, de la continuidad, lo que, para efectos de esta reflexión, supone visibilizar las prácticas que han dado lugar a la enseñanza de la biología y qué prácticas relacionadas con la pedagogía subyacen a estas, pues tales prácticas no se han mantenido iguales y se constituyen desde relaciones que son azarosas y no responden necesariamente a las lógicas hegemónicas.

De esa manera, no se pretende decir la verdad sobre la enseñanza de la biología, ni asumirla como categoría preexistente, ni campo constituido, sino como prácticas, donde las relaciones entre saberes, sujetos e instituciones posibilitan hablar de ella, y a su vez visibilizar cómo generan discursos y prácticas.

En esa perspectiva, este escrito es genealógico porque presenta, más que una universalización, preguntas sobre lo pedagógico en la historia de la enseñanza de la biología. Se ocupa de mostrar las prácticas que le han dado lugar, e incluso un no lugar, en los discursos que la constituyen. Esta problematización aporta otras miradas:"la elección del material en función de los datos del problema, focalización del análisis sobre los elementos susceptibles de resolverlo, establecimiento de las relaciones que permiten esta solución y por tanto indiferencia a la obligación de decirlo todo" (Foucault, 1971, p. 4).

Para ello, se ha conformado un archivo con documentos de diferente procedencia como libros de texto, política nacional e internacional, investigaciones de maestros, revistas de educación, artículos de periódicos, programas de formación, planes de estudio, revistas de circulación popular, entre otros, los cuales, desde los elementos de la caja de herramientas propuesta por Foucault (2002), son desarticulados en temáticas, con el propósito de poner en cuestión su lógica y sentido. Las temáticas son agrupadas en conceptos, sujetos e instituciones que posibilitan visibilizar las reglasde funcionamiento de lo que se ha denominado enseñanza de la biología y las fuerzas de poder que subyacen. Se puede rastrear qué relaciones posibilitan su emergencia, permanencia y mutación, cómo está delimitada, qué la separa, qué la entronca, los sujetos que deben y pueden hablar de ella y las instituciones que estabilizan tales modos de hablar. Esto puede identificarse en las siguientes acciones:

1. Conformación del archivo: recopilación de los documentos en distintos lugares, bibliotecas, centros de memoria, colección remota, repositorios de instituciones, páginas web, entre otras. Los documentos son registrados en una matriz según la fecha y tipo de documento.

2. Desarticulación del archivo: los documentos se tematizan. Para ello, a través de la lectura minuciosa, se extraen fragmentos que son organizados en temáticas, de acuerdo con los conceptos, las instituciones, los sujetos y las acciones, lo cual conforma la segunda matriz.

3. Lectura hipertextual: la segunda matriz es leída de distintas formas, por columnas, por filas, para visibilizarlas recurrencias, lo discontinuo, los desplazamientos, el funcionamiento. Esto da lugar a tres matrices que muestran el saber -conceptos, sujetos e instituciones-, el poder - acciones- y subjetividad - modos de

3. Asuntos como la necesidad de enseñar biología, su vínculo con la biología científica, su objeto, sus prácticas, entre otras. 
funcionamiento en el cruce de saber y poder-.

4. Escritura: desde la lectura de las matrices, se da lugar a la escritura, donde se presentan las relaciones, las prácticas, las condiciones de posibilidad, entre otras. Se propone un modo de comprender el problema, que no pretende ser la verdad sobre su inteligibilidad. El archivo es evidencia de tal comprensión.

El archivo contiene más de 900 documentos desde 1890 hasta la actualidad, lo cual no implica que la reflexión presentada responda a una linealidad de hechos. Por el contrario, presenta nodos problémicos, entendidos como cruce de relaciones que posibilitan visibilizar distintas formas y fuerzas de relación que dan lugar a las prácticas de la enseñanza de la biología. De esa manera, se pueden visibilizar las condiciones de posibilidad de su emergencia, permanencia, discontinuidad, mutación y desplazamiento, lo cual indica qué cambia y qué no se puede presentar como una totalidad. Esta investigación muestra solo una manera de leer - en la dispersión de las prácticas - lo que podría considerarse pedagógico en el devenir de la enseñanza de la biología, sus aportes y sus limitaciones.

En esa ruta de lectura y a través de la desarticulación de los textos, no se privilegia a los expertos, ni la política pública. Por ello, se incluye otro tipo de documentos que permiten visibilizar el funcionamiento de las prácticas. La noción de práctica es entendida como devenir, es decir, como singularidad a la que le subyace un campo de problemas, lo que supone pensamiento y, por ende, movimiento. Esto permite asumirla como acontecimiento. En esta reflexión, la pedagogía se configura como replantear problemas, como condición de creación y de contingencia. De ese modo, este escrito es un diagrama de las relaciones en el devenir de la enseñanza de la biología.

\section{Hallazgos. ¿Enseñanza de la biología?: una mirada desde la pedagogía}

Como diagrama, este escrito intenta visibilizar las relaciones como movimientos. En esa medida, lo que se presenta a continuación no pretende totalizar, sino proponer nodos que posibiliten cuestionamientos y reflexiones en el campo de la enseñanza de la biología desde una perspectiva contemporánea. No es una crítica o una nueva receta, sino una mirada que propone la pedagogía como posibilidad en la constitución de dicho campo, donde es posible visibilizar que la educación, el aprendizaje y la didáctica han sido protagonistas en el devenir de la enseñanza de la biología, lo cual podría sugerir que la pedagogía es asumida desde las ciencias de la educación o que la pedagogía, entendida como saber propio de los maestros, no ha tenido lugar en tal devenir. Veamos lo que muestra el archivo:

\section{La emergencia de la biología en la escuela: educar para la vida}

La biología en la escuela emerge a propósito de condiciones relacionadas con la preocupación por la vida y de los intereses sociales respecto a esta. A principios del siglo pasado, la atención hacia a la vida estaba dirigida al niño, la alimentación, el cuerpo, la enfermedad, el progreso agrícola, entre otros, los cuales en la escuela ya eran abordados desde la historia natural, la educación física, la fisiología, las ciencias de la naturaleza y la botánica. No obstante, la biología es objeto de instrucción, dado que organiza de manera distinta las relaciones entre tales preocupaciones, pues en ella confluye la noción de ciencia, que le da un estatuto diferente en lo relacionado con la verdad y la efectividad. Además, 
el concepto organismo permite acentuar la relevancia de la herencia y la evolución en el proyecto higienista de la época.

La física, la química, la biología y en general todas las ciencias basadas en la experimentación transportan en sus alas al espíritu, a la contemplación de las obras divinas y a las naciones, a las más altas cimas del progreso. (Hermano Apolinar María, 1916, p. 116)

De ese modo, es el discurso educativo - entendido como instrucción- el que confiere necesidad a la biología en la escuela, sumado al interés por alcanzar el progreso a través de la disminución de la mortalidad y de la obtención del máximo rendimiento de la población.

La enseñanza de la biología no es la reproducción de la ciencia biológica, pues, como se puede notar, se configura a partir de relaciones que responden principalmente a criterios sociales y educativos, y no a la estructura conceptual de la biología científica, debido a que está enfocada en lo humano, y desde allí en todos los saberes que estén relacionados con ello:

La escuela, en cuanto sistema social y democrático, debe educar para que los individuos y las colectividades comprendan la naturaleza compleja del ambiente, resultante de la interacción de sus aspectos biológicos, físicos, químicos, sociales, económicos y culturales; construyan valores y actitudes positivas para el mejoramiento de las interacciones hombre-sociedad naturaleza, para un manejo adecuado de los recursos naturales y para que desarrollen las competencias básicas para resolver problemas ambientales. (Ministerio de Educación Nacional [MEN], 1998, p. 23)

En consecuencia, las relaciones se transforman en el devenir de la enseñanza de la biología. Para los años 90, es visible cómo se ha instalado el discurso económico y su vínculo con el manejo de los recursos naturales. Así, la preocupación por la vida se complejiza con la noción de ambiente y con las competencias para resolver problemas. Es posible, entonces, proponer que la enseñanza de la biología ha cambiado y que su existencia en la escuela se ha transformado de acuerdo con los propósitos educativos de distintos periodos de tiempo. También se encuentra como recurrencia la vida, como objeto que le da existencia en la escuela, que no solo la distingue de la ciencia biológica, sino que la acerca a las ciencias sociales y a las ciencias de la educación como la sociología, la psicología y la economía. La vida se compone de prácticas que también cambian. De ese modo, es una de las condiciones de posibilidad de existencia de la enseñanza de la biología, lo que implica no solo permanencia; sugiere mutación y, como se muestra más adelante, desplazamiento.

\section{Hacer ciencia en la escuela: educar para la ciudadanía y la formación integral}

La enseñanza de la biología - vinculada con la ciencia biológica y con la psicología, como fuerza que determina la enseñanza - se ve reducida al aula, a lo que acontece en ella, donde se resalta el desarrollo de habilidades relacionadas con el quehacer científico, con la comprobación y la experimentación. Allí, la modificación de la conducta comienza a ser un elemento esencial.

De esa manera, se puede ver el tránsito del aula a la vida o la vida como aula, desde la perspectiva de señalar la pertinencia de la formación en ciencias, donde el ser ciudadano y su vínculo con el desarrollo le dan relevancia a la ciencia en la escuela: 
Aún es más primordial especificar para qué es relevante la ciencia escolar; por ejemplo, para la vida cotidiana, ejercer la ciudadanía, proseguir estudios posteriores, conseguir un empleo, ser científico o ingeniero, etc. La respuesta que demos a esta pregunta es también muy importante para establecer los fundamentos y el diseño del currículo escolar de ciencias y se relaciona con otra pregunta clave: ¿quién decide lo que es relevante en la ciencia escolar? (Acevedo, 2004, p. 5)

La ciudadanía, entonces, establece el deber ser de los sujetos y el fin de la educación. No obstante, las prácticas de la ciudadanía no son estáticas. Para los años 70, están vinculadas con el cuidado y con el aprecio de la naturaleza:

Todo ciudadano, como ser vivo que es, debe conocer los principios fundamentales en que se basa la vida. Con los conocimientos biológicos dados en la escuela secundaria, no podemos pretender formar científicos, pero sí ciudadanos conscientes de que la supervivencia de la vida sobre la Tierra depende en gran parte del aprecio y del cuidado que se tenga por la naturaleza. (Roldán, 1972, p. 34)

Para inicios de este siglo, la ciudadanía está ampliamente relacionada con la formación, las competencias y la innovación: "En un entorno cada vez más complejo, competitivo y cambiante, formar en ciencias significa contribuir a la formación de ciudadanos y ciudadanas, capaces de razonar, debatir, producir, convivir y desarrollar al máximo su potencial creativo" (MEN, 2006, p. 6).

Así, hacer ciencia en la escuela está relacionado con la formación del ciudadano, desde una perspectiva integral que favorezca el desarrollo sostenible y la calidad de vida, los cuales responden principalmente a criterios económicos. Se prioriza un cambio de conducta en cuanto a la salud, el emprendimiento y el ambiente, lo que también sugiere el desplazamiento de la enseñanza al aprendizaje.

\section{El simulacro de la biología científica}

Las prácticas rastreadas visibilizan que la enseñanza de la biología se relaciona con la vida y los modos de regulación de esta, que van cambiando según las condiciones de diferentes periodos históricos. El estatuto de cientificidad le es otorgado por la "biología científica", lo cual le da veracidad y relevancia. No obstante, como ya se ha mostrado, esto no es la reproducción de dicha ciencia, pues en la enseñanza se construyen relaciones distintas, e incluso se omiten conceptos que pueden ser estructurantes para la biología científica.

La experimentación como una estrategia didáctica implica que los estudiantes normalistas sepan identificar problemas y proponer formas de resolverlos. Se debe buscar que las actividades que realicen durante este semestre promuevan la curiosidad y desarrollen las habilidades, las actitudes y los valores relacionados con la actividad científica, de tal manera que la experimentación contribuya a la formación integral del alumno normalista, que posteriormente pueda ser desarrollada en la escuela primaria. (Secretaría de Educación Pública, 2005, p. 11)

La experimentación, como rasgo distintivo de las ciencias, es considerada como estrategia didáctica para su enseñanza. La investigación escolar es otra forma de posibilitar la resolución de problemas y la innovación. Así, se mantiene la ficción -el simulacronombrada por Álvarez (2003), quien señala quela enseñanza dela biología no es reproducción 
de los conocimientos científicos, pero sí está relacionada con ellos -en tanto condición de posibilidad de existencia- desde la noción de verdad y como forma de constituir un objeto de enseñanza validado por las disciplinas. Esto permite interrogar por el objeto de la enseñanza de la biología, que, por supuesto, no es el de la disciplina científica.

\section{Enseñanza de la biología y las ciencias naturales: entre permanencias y mutaciones}

Al rastrear el devenir de la enseñanza de la biología en la escuela colombiana, se encuentra un vínculo muy estrecho con las ciencias naturales, las cuales también han recorrido su camino en condiciones singulares. Así, es posible enunciar algunas de sus transiciones, donde se encuentra como recurrencia el estudio de la naturaleza y su configuración a partir de ciencias que "estudian la vida". De esa manera, a finales de 1890, es posible distinguir las ciencias de la naturaleza basadas en la observación y en los cuerpos naturales, mientras que, a mediados de la década de los 30 , están relacionadas con la contemplación de la naturaleza y el uso de los recursos:

Estudios de la Naturaleza. Tiene por objeto hacer que el niño comprenda por medio de la observación directa y de la experimentación posible, las causas de los principales fenómenos naturales que ocurren en la vida diaria [...] Interesan mucho las nociones acerca de la vida de animales y plantas más comunes, y de las propiedades de los minerales utilizados en la industria. (MEN, 1935, p. 91)

En la década de los 50, se agrupan la física, la química y la biología: "Las ciencias naturales investigan en tres grandes conjuntos, naturaleza física, naturaleza química, naturaleza biológica" (Recanses, 1948, p. 9). La agrupación también ha sufrido mutaciones a propósito de las nociones de integración y de interdisciplinariedad. Esta última establece las relaciones con tecnología y sociedad:

Destacar la importancia de la enseñanza de las ciencias y de la cultura tecnológica en toda la educación básica como elemento importante de la educación para la vida, vinculándola, por una parte, a la idea del mantenimiento de un ambiente sustentable y, por otra, insistiendo en su uso para el desarrollo del pensamiento lógico en relación con la búsqueda de la información y con la solución de problemas prácticos. (Unesco/Orealc, 1991, p. 41)

Esta relación, vinculada con la noción de desarrollo y aprendizaje, ha dado lugar al planteamiento de los conocimientos pertinentes a propósito de la autosostenibilidad y la sustentabilidad, y es condición de posibilidad para el desplazamiento de la biología en la escuela colombiana. Tal desplazamiento se visibiliza no solo por su ausencia en el plan de estudios o por el cierre de los programas de formación universitarios, sino también porque desde la pertinencia, el saber sobre lo vivo desde la vida es desplazado por una mirada a la vida desde la empresa y el aprendizaje. No obstante, ese desplazamiento no implica inexistencia, pero sí invisibilización y la irrupción de otras formas, como la biopedagogía.

\section{La ausencia de la pedagogía: del método a la biopedagogía}

Como se ha podido ver hasta aquí, la pedagogía es poco nombrada, y, cuando aparece, está relacionada con el hacer y no con el pensar, pues, al parecer, lo que se debe pensar viene dado por la educación y las ciencias que deben proponer tales pensamientos. Es muy frecuente la relación educación-enseñanza, donde esta última queda subordinada a las demandas de la primera. 
Programa de ciencias pedagógicas. Pedagogía general y legislación escolar para $\mathrm{V}$ año. 1 . En qué consiste el proceso de la educación. 2. Factores que contribuyeron a la educación. 3. Educación integral del niño. 4. Escuela activa y escuela pasiva. 5. El programa escolar. 6. Espíritu y contenido elemental. 7. Asignaturas que debe contener el programa. 8. El medio ambiente como tema de estudio en la escuela pública. 9. El principio de globalización en el desarrollo del programa. 10. Un programa de centros de interés. 11. Principios generales de enseñanza. 12. Métodos de educación. (Arciniegas, 1946, p. 56)

La enseñanza es objetivada a través de la planificación y el método, pero al tiempo se encuentra en la tensión entre los intereses y el desarrollo social. Desde esa perspectiva, el maestro es capacitado para optimizar y hacer más eficiente la enseñanza.

Centro de Estudios Pedagógicos. Atendiendo a las órdenes emanadas del ministerio, de la dirección y de la inspección de educación, fueron establecidos y funcionaron normalmente desde el principio del año en curso estas instituciones de carácter científico y social, en donde los maestros interesados por la obra educadora venían en pos de su perfeccionamiento intelectual y técnico pedagógico que los capacitara para dar a sus discípulos una enseñanza más eficiente y racional. (J. Medina, 1941, p. 17)

No obstante, como lo han señalado otros autores (Quiceno, 2010), el maestro ha sido desplazado. La función docente - junto con la profesionalización y la capacitación- ha dado lugar al docente como un "cualquiera" que administra y gestiona el conocimiento. En el devenir de la enseñanza de la biología, se encuentra otra noción: "mediador", que tiene lugar en el cruce de relaciones entre el aprendizaje y la vida, donde la resolución de problemas es el lugar de la pedagogía:

Los mediadores podamos entender cómo resolver situaciones a través de vías alternativas. Es decir, el camino que debe seguir un mediador en los procesos de aprendizaje es la mediación pedagógica y la transformación de los procesos desde la complejidad. He aquí la pasión pedagógica, el ambiente pedagógico, el umbral pedagógico, la madurez pedagógica, la certidumbre pedagógica y el desarrollo próximo. (Devia, 2018, p. 187)

Así, basada en otras disciplinas, la pedagogía adquiere una existencia nominal, que apellida a cualquier asunto que deba tener un remedio relacionado con la emocionalidad y el hacer, donde el aprendizaje posibilita el buen vivir:

De lo simple a lo complejo, empecemos por observar que la palabra biopedagogía está compuesta por dos partes: bio, que etimológicamente remite a la palabra vida, y pedagogía, la cual delinea los procesos de aprendizaje en el ser humano. Así la biopedagogía implica educación para la vida, para el mejor vivir de todas sus facetas y lugares. (Devia, 2018, p. 182)

La biopedagogía es posiblemente otra forma de existencia en el devenir de la enseñanza de la biología, la cual, al trascender el aula o al entenderla vida como aula, estabiliza la relación entre vida y aprendizaje, donde la enseñanza y la pedagogía por la vía del pensamiento están ausentes y cobran existencia desde el amor, la emoción y la ternura. Así, su fuerza es en y para toda la vida. 


\section{Las mutaciones de la enseñanza: calidad de vida}

La enseñanza, relacionada con la verificación del método, le ha dado existencia a la evaluación, la cual dejó de estar centrada en los conocimientos o en el quehacer del maestro. Con el aprendizaje significativo y permanente, oficializa la función docente y el desempeño como habilidades para la solución de problemas, lo cual se justifica en la calidad y en la necesidad del aprendizaje. Allí, lo pedagógico está centrado en el manejo de lo que acontece en el aula.

La enseñanza ha tenido un vínculo muy estrecho con la psicología como una de las ciencias de la educación. En este sentido, las prácticas han estado relacionadas con normalizar y modificar las conductas:

Como muchos otros de los principios que informan la labor educativa, este movimiento saludable ha tenido su origen en los pueblos del Norte; hoy todo sistema que aspire a ser completo y racional debe profesar un triple objeto: la educación del cuerpo, de la inteligencia y de la voluntad. (Montoya \& Manrique, 1913, p. 460)

El aprendizaje, como su pareja durante mucho tiempo, le dio mayor fuerza a dicha tarea. No obstante, la transición de la escuela expansiva a la escuela competitiva (Martínez, 2004) la ha venido desplazando, pues es posible aprender en todo lugar, en una sociedad que privilegia la velocidad y utilidad de la información, en la necesidad de aprender para toda la vida y ser productivo.

Las nuevas expectativas sociales exigían que el sistema educativo fuera más allá de garantizar escolaridad universal, y ofreciera a todos los estudiantes, independientemente de su procedencia, oportunidades para desarrollar las habilidades y valores necesarios para vivir, convivir, ser productivo y seguir aprendiendo a lo largo de la vida (MEN, 2006, p. 8)

La relación aprendizaje-vida denota una mutación de gran relevancia para la enseñanza de la biología, pues la vida misma es objeto de modulación ${ }^{4}$ de conductas, donde la ficción de la ciencia biológica es insuficiente, pues las relaciones con el ambiente, el emprenderismo y la autogestión son estabilizadas en la formación en ciencias naturales y educación ambiental. La vida, desde la calidad de la educación, posibilita estabilizar lo que es necesario para aprender, pues ya no es relevante el aula, el maestro o el método, sino la formulación de proyectos de vida, experiencias exitosas, ambientes de aprendizaje y autogestión, que permitanobjetivizar el buen vivir.

\section{La biopedagogía: otro modo de existencia del aprendizaje}

"Aprender, mantenerse en un estado de aprendiencia (sic) permanente, se refiere a establecer la telaraña de la vida: una red o trama de interacciones neuronales muy complejas y dinámicas que van creando estados generales cualitativamente nuevos en el cerebro humano" (Villareal, 2011, p. 4).

4. Se propone modulación porque el rastreo realizado muestra que las formas de saber, las acciones y los sujetos relacionados con ella no se configuran exclusivamente desde el disciplinamiento o el control, donde el aprendizaje es quizás uno de los mecanismos para ello. Así, la modulación a través del aprendizaje - entendido como mecanismo de hacer ver y pensar-emplea técnicas de gestión y digitalización, que posibilitan otras formas de tiempo, "la vida en otro tiempo", la vida que se configura en "estilos" y en un "buen vivir" a través de la hiperrealización de las emociones y el "amor", los cuales fluyen y son codificados en redes a través del registro y la selección de datos. 
A través de la biopedagogía se formaliza la relación aprendizaje-autogestión. De ese modo, a través de conceptos biológicos como el de autopoiesis, se pretende regular las conductas, ya no solo por medio de comportamientos, sino por medio del control cerebral.

Por ello, serán aprendientes todos aquellos agentes cognitivos (personas, grupos, organizaciones, instituciones, sistemas) que llevan a cabo experiencias de aprendizaje desde sus vidas, mediante esa permanente autoorganización que entreteje la compleja red de interacciones de la vida en su totalidad. De allí que principalmente en Europa, el término "sociedades aprendientes" se ha convertido en una expresión de mucho y frecuente uso para cubrir el aprendizaje durante la vida (life-long-learning), concepto que incluye todo tipo de participación activa en procesos cognitivos, desde la célula viva hasta los procesos socioculturales. Todo ser vivo está confrontado con un "life-long-learning". (Villareal, 2011, p. 5)

De ese modo, se naturaliza el aprendizaje permanente, se le da una existencia vital y se readecúa la noción de sistema, desde las experiencias y los ambientes para el aprendizaje:

En otros términos, en el proceso generador de la vida, el aprendizaje configura la actividad vital de los organismos biológicos; de ahí que la existencia de la cognición es la existencia vital. Así, todo ser vivo tiene la necesidad de aprendizaje de forma auténtica y genuina, conservando, de manera flexible y adaptativa, la dinámica de permanecer aprendiendo, por lo que Assmann identifica que los procesos vitales y los procesos de conocimiento son una misma instancia. (R. Medina, 2017, p. 50)

La vida, entonces, es autoorganización, autogestión y sostenibilidad. Así, se vincula la vida y el aprendizaje, no solo desde conceptos biológicos, sino también desde la emocionalidad y la incertidumbre:

La biopedagogía concibe el aprendizaje como la propiedad que tienen todos los seres vivos para autoorganizar la vida. El aprendizaje como un proceso de autoorganización de la vida, obedece a los mismos procesos que sustenta el paradigma emergente: autoorganización, incertidumbre y sostenibilidad. Veamos: el proceso de vivir es un proceso de cognición y el conocer es uno de los elementos esenciales que hace posible la autoconstrucción de los seres vivos. (Gutiérrez, 2010, p. 2)

Así, se podría pensar que la biopedagogía no es enseñanza de la biología, porque, aunque vincula conceptos biológicos, las relaciones que establece son distintas y formaliza la idea de la pedagogía como aprendizaje; o en otra lectura, es una irrupción en la enseñanza de la biología, que visibiliza su desplazamiento — sospechas que están por indagar, dada su reciente emergencia-.

Así como propiciar el avance hacia la tercera cultura y una episteme humanizadora que permita la vinculación del conocimiento a la vida por medio de la percepción del ser vivo, es decir, donde se pueda sentir, comunicar, razonar y construir un mundo posible para todos. El desafío de la biopedagogía es alcanzar, a través de la biología del amor y la ternura, procesos de aprendizaje complejos, holísticos, legítimos y autoorganizados. (Devia, 2018, p. 179)

En consecuencia, la biopedagogía es toda una ruta de indagación y problematización, pues, una vez más, es posible ver cómo el aprendizaje va ganando lugar en todos los espacios, 
no solo en las ciencias, también en la educación, en la economía... Es una gran fuerza que despliega un sinnúmero de prácticas que, desde una perspectiva contemporánea, posibilita ver el movimiento de las distintas formas de producción de la vida. Esto permite formular las preguntas por cómo el aprendizaje se ha constituido en una fuerza articuladora, cómo la vida se constituye en un problema - en una preocupación recurrente- y a partir de cuándo y cómo el aprendizaje se convierte en un modo de articulación de las prácticas relacionadas con la vida.

\section{Conclusiones: la investigación continúa}

La mirada a la configuración de la enseñanza de la biología, aunque no pretende ser una totalización y otra forma de verdad, posibilita hacer afirmaciones a modo de cuestionamientos, que son una apertura para continuar la investigación, pero también para, desde la pedagogía, problematizar el campo de la enseñanza de la biología y la formación de maestros.

Desde los planteamientos de Zuluaga et al. (2003), en el rastreo realizado se encuentra la pedagogía como si fuera lo mismo que hablar de educación. La pedagogía está cooptada por las ciencias de la educación, donde la enseñanza es asumida desde la técnica y el método.

En esa dirección, parece que, en el campo de la enseñanza de la biología, la discusión de la enseñanza está reducida a la reproducción de la ciencia, lo que es validado por apropiaciones de la didáctica asociadas a la verificación de conceptos, al desarrollo de competencias y a la transposición de la disciplina científica.

La vida y sus prácticas le han dado un modo de existencia a la biología en la escuela, que cambia según condiciones relacionadas con la educación y la economía. En este sentido, puede visibilizarse que ha estado relacionada con la higiene, la alimentación, la salud, el cuerpo, la educación sexual (Roa, 2017) y el ambiente; donde se privilegia la preocupación por lo humano. De ese modo, no es una reproducción de la estructura conceptual de la biología científica.

Entonces, ¿cuál es el objeto de la enseñanza de la biología? Desde una perspectiva contemporánea, se afirma que las relaciones entre la vida y lo vivo. No obstante, estas relaciones, fundadas desde las ciencias de la educación, el aprendizaje, la educación ambiental y la interculturalidad, son vistas como condiciones que posibilitan su desplazamiento.

La biopedagogía como otra forma de existencia visibiliza las relaciones aprendizaje-vida desde una perspectiva del control cerebral, que no requiere ser asignaturizada, ni objeto de formación, ya que es natural de los seres vivos, y todos están en capacidad de autogestionar su mantenimiento y producción como aprendientes, para lo cual no se requiere escuela, ni maestros.

\section{Referencias}

Acevedo, D. (2004). Reflexiones sobre las finalidades de la enseñanza de las ciencias: educación científica para la ciudadanía. Revista Eureka sobre Enseñanza y Divulgación de las Ciencias, 1(1).

Álvarez, A. (2003). La pedagogía y las ciencias: historias de una relación. En O.Zuluaga, A. Echeverri, A. Martínez, H. Quiceno, J. Sáenz, \& A. Álvarez, Pedagogía y Epistemología. Magisterio. 
Arciniegas, G. (1946). Escuelas Normales regulares. Plan de estudios, programas, reglamentos $y$ disposiciones vigentes. Imprenta Nacional.

Avanzini, G. (1990). La pedagogía desde el siglo XVII hasta nuestros días. Fondo de Cultura Económica.

Chona, G., Castaño, N., Cabrera, F., Arteta, J., Valencia, S., \& Bonilla, P. (1998). Lo que nos dice la historia de la enseñanza de la biología en Colombia — una aproximación-. Tecné, episteme y didaxis: TED, (4). https://doi.org/10.17227/ted.num4-5686

Devia, J. (2018). La biopedagogía: una mirada reflexiva en los procesos de aprendizaje. Praxis \& Saber,9(21), 179-196. https://doi.org/10.19053/22160159.v9.n21.2018.7862

Foucault, M. (1971). El polvo y la nube.En La imposible prisión: Debate con Michel Foucault. Anagrama.

Foucault, M. (2002). La arqueología del saber. Siglo XXI Editores.

Goodson, I. (2008). Procesos sociohistóricos de cambio curricular. En A.Benavot\&C. Braslavsky. El conocimiento escolar en perspectiva histórica y comparativa. Cambios de currículos en la educación primaria y secundaria (pp. 335-350). Granica.

Gutiérrez, F. (2010). Las nuevas ciencias de la vida. Polis, Revista Latinoamericana, (25). https://journals.openedition.org/polis/423

Hermano Apolinar María. (febrero, 1916). Boletín de la sociedad de Ciencias Naturales del Instituto de la Salle, 4(26).

Marín, D. (2019). Autoayuda, educación y prácticas de sí. Genealogía de una antropotécnica. En A. Álvarez, B. Barragán, J. Echeverri, J. Fayad, D. Marín, A. Martínez, C. Noguera, J. Orozco, D. Rubio, \& J. Sáenz, Genealogías de la Pedagogía. Universidad Pedagógica Nacional.

Martínez, A. (2004). De la escuela expansiva a la escuela competitiva. Dos modos de modernización en América Latina. Anthropos.

Medina, J. (1941). Educación en Boyacá. Secretaría de Educación. Inspección de la Escuela Primaria. Imprenta Nacional.

Medina, R. (2017). Bioaprendizaje y educación intercultural. Sophia, 13(1), 47-54. http:// www.scielo.org.co/pdf/sph/v13n1/v13n1a06.pdf

Ministerio de Educación Nacional. (1935). El texto de los programas de Primera y de Segunda Enseñanza. Imprenta Nacional.

Ministerio de Educación Nacional. (1998). Lineamientos curriculares. Ciencias Naturales y Educación Ambiental. http://menweb.mineducacion.gov.co/lineamientos/ciencias/ ciencias.pdf

Ministerio de Educación Nacional. (2006). Estándares básicos de competencias en lenguaje, matemáticas, ciencias y ciudadanas. https://www.mineducacion.gov.co/1621/ articles-340021_recurso_1.pdf

Montoya, J. \& Manrique, J. (1913). Repertorio de medicina y cirugía, 4(9). Imprenta Nacional. 
Not, L. (2000). Las pedagogías del conocimiento. Fondo de Cultura Económica.

Quiceno, H. (2010). El maestro, el docente y el formador. En Figuras Contemporáneas del Maestro en América Latina. Magisterio.

Recanses, J. (1948). Ciencias naturales básicas. Voluntad.

Riveros, O. (2006). Historia de las prácticas de enseñanza de la biología y la pedagogía en el Departamento de Biología de la Universidad Pedagógica Nacional. Informe de investigación. CIUP - UPN.

Roa, P. (2017). Salud, cuerpo y vida: una genealogía de la educación sexual en la escuela colombiana. Praxis \& Saber, 8(17), 67-84. https://doi.org/10.19053/22160159. v8.n17.2018.4714

Roa, P. \& Herrera, J. (2010). Historia de los saberes escolares: la emergencia de la biología en la escuela colombiana 1900-1930 (Trabajo de grado, Maestría en Educación). Universidad Pedagógica Nacional.

Roa, P., Jaramillo. C., Serrato, D., \& Sánchez, L. (2018). Investigación, maestro e historia: la biología como saber escolar ( $1^{\text {a }} \mathrm{ed}$.). CIUP - UPN.

Roldán, G. (1972). La enseñanza de la biología. Metodología de la enseñanza de la biología. Actualidades biológicas, 1(2).

Secretaría de Educación Pública. (2005). Ciencias Naturales y su Enseñanza II. Programa y materiales de apoyo para el estudio. http://www.enesonora.edu.mx/plan_estudios/ programas/5lep/cnat2.pdf

Unesco/Orealc. (1991). Proyecto principal de educación en América Latina y el Caribe. Boletín No 24.

Universidad Pedagógica Nacional. (1984). Programa de Licenciatura en Biología. Nuevo plan de estudios. Facultad de Ciencia y Tecnología, Departamento de Biología.

Villareal, A. (2011). Una pedagogía para la vida. Ponencia presentada en el II Congreso Internacional de Investigación Educativa 2011 en la Universidad de Costa Rica. https://www.uv.mx/veracruz/cosustentaver/files/2015/09/19.Villareal-A.M-2011_Unapedagogia-para-la-vida.pdf

Zuluaga, O. (1999). Pedagogía e Historia: La historicidad de la pedagogía: la enseñanza, un objeto de saber. Editorial Universidad de Antioquia, Anthropos, Siglo del Hombre editores.

Zuluaga, O., Echeverri, A., Martínez, A., Quiceno, H., Sáenz, J., \& Álvarez, A. (2003). Pedagogía y epistemología. Magisterio. 